\title{
Que disent les tapirs? De la communication avec les non-humains en Amazonie
}

Cédric Yvinec

\section{OpenEdition}

Journals

Édition électronique

URL : https://journals.openedition.org/jsa/2811

DOI : 10.4000/jsa.2811

ISSN : 1957-7842

Éditeur

Société des américanistes

Édition imprimée

Date de publication : 5 janvier 2005

Pagination : 41-70

ISSN : 0037-9174

\section{Référence électronique}

Cédric Yvinec, "Que disent les tapirs ? De la communication avec les non-humains en Amazonie », Journal de la Société des américanistes [En ligne], 91-1 | 2005, mis en ligne le 10 janvier 2010, consulté le 04 septembre 2022. URL : http://journals.openedition.org/jsa/2811 ; DOI : https://doi.org/10.4000/ jsa.2811 


\title{
QUE DISENT LES TAPIRS ? \\ DE LA COMMUNICATION AVEC LES NON-HUMAINS EN AMAZONIE
}

\author{
Cédric YVINEC *
}

\begin{abstract}
S'appuyant sur une comparaison de divers faits de communication avec des entités non humaines (animaux, esprits, morts, etc.) chez les Indiens des Basses Terres de l'Amérique du Sud, cet article a pour but de montrer comment ces événements, en dépit des apparences, relèvent d'un même principe général d'interprétation et utilisent un même langage fondé sur la parenté. [Mots clés : Amazonie, animaux, morts, esprits, rêve, communication, cosmologie.]
\end{abstract}

What do the tapirs say? About communication with non-human beings in Amazonia. This article compares different communicational events with non-human beings (animals, spirits, dead, etc.) among Lowland Indians from South America. These facts reveal to be the matter for the same general interpretation ; they use the same language based on kinship. [Key words: Amazonia, animals, dead, spirits, dream, communication, cosmology.]

¿Qué dicen los tapires? Acerca de la comunicación con entidades no-humanas en Amazonía. Al comparar varios hechos de comunicación con entidades no-humanas (animales, espíritus, muertos, etc.) entre los indios de las tierras bajas de la América del Sur, intentamos demostrar cómo esos sucesos, a pesar de las apariencias, remiten a un mismo principio general de interpretación y utilizan un mismo lenguaje basado en el parentesco. [Palabras claves : Amazonía, animales, muertos, espíritus, sueños, comunicación, cosmología.]

Les Indiens des Basses Terres prétendent parler à des interlocuteurs que nous sommes contraints de regrouper sous le terme disgracieux de « non-humains » : il s'agit de végétaux, animaux, esprits, défunts, cailloux ou objets manufacturés... conversation qui ne diffère guère, de leur point de vue, de celle entretenue avec un étranger; de surcroît s'il est blanc. Ces croyances s'intègrent logiquement dans les

* EHESS, Paris [cedric.yvinec@ens.fr].

Journal de la Société des Américanistes, 2005, 91-1, pp. 41-70. O Société des Américanistes. 
descriptions actuelles des ontologies amérindiennes - « animisme », " perspectivisme »-, puisque tout être est susceptible d'apparaître comme humain dans certaines circonstances. Mais leurs diverses formes et modalités n'ont pas fait l'objet d'études détaillées et systématiques, peut-être parce que, aux yeux des ethnologues, elles relèvent de domaines multiples, allant des rêves et des chants chamaniques à la crainte des fantômes, en passant par des rituels guerriers ou cynégétiques. Ces faits correspondent pourtant à une des principales manifestations « concrètes » des ontologies sous-jacentes. En dépit de l'hétérogénéité de ces manifestations, les Indiens leur attribuent implicitement un caractère commun : elles sont considérées comme un champ de communication alternatif à celui de la communication humaine. Plus on parle avec les animaux - ou plus on parle de manière animale - et plus on les écoute, moins on sera capable d'entendre les humains - c'est-à-dire les « vrais » humains, les parents - et de parler avec eux : à chaque « dialogue » non-humain, on risque la surdité, le mutisme, la cécité, voire la mort.

Une étude complète - qui devrait prendre en compte les modalités sensorielles et/ou linguistiques de ces faits, leurs contextes d'émergence, leurs conditions de narration, de représentation et de crédibilité, leur usage social, etc. - n'est pas envisageable ici. Nous proposons plutôt une analyse de leurs dimensions pragmatiques et sémantiques à partir d'exemples provenant d'une quarantaine de groupes amazoniens. D'emblée, il convient d'écarter, ou du moins de limiter, l'importance d'une notion qui est introduite implicitement dans nombre de descriptions ethnographiques : celle d'un « code » d'interprétation des messages non-humains. Cette notion n'est certes pas toujours dénuée de pertinence, notamment dans les cas où le sens d'une interaction avec un non-humain est décidé en fonction de règles plus ou moins explicites, déjà bien connues du sujet (ou de ceux qui savent la décoder pour lui). Il s'agit aussi bien des interprétations fixes de tel ou tel élément - en particulier dans des interactions très ritualisées, comme certaines initiations au chamanisme ou quêtes d'auxiliaires (Wagley 1977 ; Crocker 1985 ; Surrallés 2003) - que des significations construites par l'application de règles générales - comme pour les oniromancies fondées sur des logiques d'inversion, assez semblables à celles mises en évidence dans la mythologie analysée par Lévi-Strauss (Guss 1980 ; Descola 1989). Mais, dans ces cas, les éléments signifiants et le " code » pertinent sont toujours le produit d'une sélection, opérée au sein d'un contexte offrant plusieurs significations potentielles plus ou moins pertinentes et cohérentes, plus ou moins mutuellement exclusives, chaque interprétation d'un événement nourrissant les suivantes et modifiant en retour les précédentes (pour un exemple précis, voir Surrallés 2003, pp. 239-240). L’interprétation de ces interactions - incluant donc le choix de ces codes - serait par conséquent mieux décrite par un modèle "inférentiel », au sens d'un processus dynamique, s'alimentant de lui-même et régi par un " principe de pertinence » (Sperber et Wilson 1989). 
Signe OU ACTE?

Plus fondamentalement, la notion d'interprétation semble poser problème dans le cas de ces interactions communicationnelles avec des non-humains. Cette difficulté surgit en particulier dans l'étude des rêves amazoniens. On trouve en effet dans nombre de groupes une distinction entre des rêves qui nécessitent une interprétation et ceux qui n'en ont aucunement besoin. Ces derniers sont conçus comme des événements complets, se suffisant à eux-mêmes, dans lesquels les faits n'ont pas d'autre sens que celui qu'ils auraient en état de veille et où les dialogues correspondent précisément à leur contenu explicite. Si ces rêves n'ont pas à être interprétés, c'est qu'ils ne sont pas des signes, renvoyant à autre chose qu'à eux-mêmes. Ces communications sont des actes au même titre que les dialogues entre humains éveillés. Certes, comme ces derniers, elles peuvent « signifier » plus que le contenu apparent du dialogue - par exemple, l'établissement d'une relation d'amitié ou d'alliance entre les interlocuteurs. Mais ce sens profond, tout comme le sens apparent, n'est pas différent de celui que pourrait avoir un dialogue exactement semblable en état de veille. Les ethnographes reconnaissent donc souvent une distinction entre deux grandes catégories de rêves, habituellement nommées «métaphoriques » et "littéraux ». Cette distinction existe dans de nombreux groupes : les Achuar la soulignent explicitement par une distinction terminologique; les Kalapalo et les Parintintin la marquent de manière plus implicite par une distinction entre deux étiologies du rêve, voyage de l'âme et rencontre d'esprit ; les Suya et les Katukina semblent l'utiliser sans la théoriser (Descola 1989 ; Basso 1987 ; Kracke 1990 ; Seeger 1981, p. 222 ; Coffaci de Lima 2000, pp. 87, 133). Plus généralement, en dépit des lacunes ethnographiques, on peut supposer qu'elle se trouve implicitement partout où se rencontrent simultanément des interprétations fondées sur des inversions - donc des rêves « métaphoriques ", signifiant ou renvoyant à autre chose qu'à leur contenu propre - et des obtentions, des transmissions ou des apprentissages en rêve - de chants, de messages ou de motifs divers - et/ou des risques de séduction par des esprits malveillants - entraînant maladies et mort, etc. Rares sont les groupes des Basses Terres chez lesquels on ne trouve pas, d'une manière ou d'une autre, ces deux classes de rêves ${ }^{1}$.

Cependant, la généralisation de cette taxinomie semble problématique, car il n'est pas évident de découvrir un critère récurrent permettant de distinguer concrètement ces deux classes. Si, chez les Achuar, c'est la présence d'un individu connu dans le rêve qui déclenche une interprétation littérale, cela n'est pas vrai chez les Apinayé, pour qui rêver de se disputer avec son père annonce une querelle avec un autre individu. De plus, une telle " dispute » constitue probablement aussi une interaction sonore et linguistique. Ce caractère ne suffit donc pas non plus à justifier une interprétation littérale - par opposition aux rêves métaphoriques qui seraient essentiellement visuels - comme il semble que ce soit le cas chez 
les Achuar et les Kalapalo (Descola 1993 ; Da Matta 1970 ; Basso 1987). Une autre hypothèse a été suggérée par Basso (ibid.) : il y aurait une corrélation entre le caractère iconique ou indiciaire et la relation au langage. Chez les Kalapalo, en effet, ce serait le fait que certains rêves - notamment les rêves " prophétiques 》 soient conçus comme servant à fixer une intention et un but à l'action, qui impliquerait qu'ils soient racontés, discutés et, par conséquent, considérés comme métaphoriques pour pouvoir être manipulés ${ }^{2}$. Malheureusement, cette corrélation est loin de se retrouver dans tous les cas : chez les Achuar, les rêves kuntuknar, augures métaphoriques de succès cynégétiques, sont souvent gardés secrets ou révélés dans un cercle très restreint, entre époux par exemple; en revanche, ils discutent les rêves mesekrampar, présages funestes mais tout aussi métaphoriques, ainsi que la plupart des rêves karampar, conçus comme des interactions littérales avec d'autres êtres, humains ou non.

Remarquons que les rêves à interprétation littérale, par opposition à ceux qui nécessitent une interprétation métaphorique, ont un "résultat» immédiat puisqu'ils permettent d'obtenir un instrument « utilisable » sans délai. L'exemple classique est celui de l'obtention d'un « chant ». C'est le cas chez les Suya où les chants sont littéralement entendus, par opposition aux présages métaphoriques ; chez les Katukina où les connaissances et les chants thérapeutiques sont aussi transmis par une interaction linguistique littérale, au contraire des présages de mort codés ; chez les Achuar qui obtiennent, outre des spectres arutam - du moins pour les femmes -, des chants anent et des charmes par les karampar littéraux (Seeger 1981, p. 219 ; Coflaci de Lima 2000 ; Descola 1989). Il s'agit parfois d'objets moins " concrets » que des chants, par exemple des identités : chez les Parintintin, un chamane peut susciter, en rêve, la naissance d'un successeur qui sera une incarnation d'esprit. Cette croyance se retrouve dans de nombreux groupes, depuis les Kaingang jusqu'aux Shipibo, en passant par les Tapirapé (Kracke 1990; Henry 1964, p. 73 ; Saladin d'Anglure et Morin 1998; Wagley 1977, p. 134). Les rêves métaphoriques sont, pour leur part, en général décrits comme des " présages », donc par définition sans résultat immédiat.

Toutefois, pour ne pas rester à un niveau qui peut paraître légèrement tautologique, il convient d'approfondir et de préciser cette idée : les Achuar nomment le rêve littéral penke karampar, "véritable rêve " (Descola 1993, p. 133), ce qui peut sous-entendre que les rêves nécessitant une interprétation sont moins « véritables » et donc, peut-être, inachevés en tant qu'interaction - seul le karampar est une véritable communication entre le rêveur et les individus rêvés, les deux autres formes étant probablement des rêves et des actes inaboutis ou embryonnaires. Ce dernier point soulève un autre problème : l'inadéquation de la notion de " présage », souvent utilisée pour qualifier les rêves métaphoriques. Comme le souligne Surrallés (2003, p. 192), il faut voir dans les rêves, métaphoriques ou littéraux, comme dans la plupart des dialogues non humains, moins des prédictions ou des assurances sur un avenir en quelque sorte déjà écrit de manière linéaire, que des 
actions sur la volonté ou un " travail sur la subjectivité ». Avant la guerre ou la chasse, il est nécessaire de se forger une intentionnalité prédatrice - un « cœur de jaguar » en termes candoshi. Le succès cynégétique ou guerrier n'est rien d'autre que le plein accomplissement de cette subjectivité, c'est-à-dire l'actualisation d'une relation « jaguaresque » à tel ou tel objet. Mais, dès lors, on voit vraiment les choses comme elles sont en tant que termes de cette relation enfin stabilisée. On les voit « littéralement » : par exemple, le guerrier candoshi, devenu pleinement jaguar - i.e. meurtrier -, mord «littéralement» sa victime. Réciproquement, lorsqu'on ne voit pas les choses comme elles «sont » vraiment, c'est que la relation n'est pas actualisée sous sa forme définitive, qu'elle est encore instable, qu'il y a un écart entre la relation telle qu'elle est au moment du rêve et telle qu'elle sera lorsqu'elle aura atteint l'état vers lequel elle semble tendre. Pour le dire succinctement, les "présages " n'annoncent pas le futur, ils ne sont que des symptômes du présent. Ils ne sont pas un discours prophétique, fondé sur une vision par anticipation d'un état qui n'existe pas encore, ils ne sont qu'une perception plus fine et plus lucide d'un état actuel. On peut donner diverses confirmations de cette assertion. Au niveau des croyances explicites d'abord : le " télescope chamanique » parakanã, par exemple, permet de voir ce que font actuellement les ennemis - et éventuellement d'en déduire leurs intentions -, et non pas ce qu'ils feront (Fausto 2001, p. 285). Au niveau des indices linguistiques ensuite : en araweté, " rêver » se dit, entre autres, au moyen de l'aspect verbal de la potentialité non réalisée (Viveiros de Castro 1992, p. 367). Enfin, au niveau de la « logique » des pratiques : s'il s'agissait de « présages », d'annonces du futur, on tenterait de les vérifier; d'évaluer la certitude de leur prédiction. Ce fut la tendance spontanée de certains ethnologues qui croyaient voir une ruse de charlatan dans le fait que la prédiction était valable indéfiniment et finissait donc par se réaliser (Henry 1964, p. 90). Mais, si cette validité est indéfinie, c'est précisément parce qu'il ne s'agit pas d'une prédiction annonçant un événement à un jour dit, mais d'un diagnostic de l'état présent, assorti d'une spéculation sur son évolution probable ; or le fait qu'un symptôme d'une maladie ne permet pas de prédire la mort ou la guérison à un moment précis, mais de donner une tendance de l'état de santé, n'a pour nous rien de faux, ni de charlatanesque. Selon Lima (1996), si après un " mauvais présage », annonçant par exemple une attaque d'ennemi, on reste chez soi, ce n'est pas pour éviter un lieu « prédit »-ce qui serait d'ailleurs absurde, puisqu'il semble plus probable de rencontrer l'ennemi chez soi qu'en forêt -, mais pour ne pas se retrouver en un lieu générique - comme la forêt - qui est celui d'un affaiblissement relatif - i.e. d'une désocialisation -, et qui contribuerait donc à aggraver les symptômes. De plus, s'il s'agissait de présages, on ne chercherait pas à favoriser intentionnellement et explicitement leur venue ou à l'empêcher, ni à les faire se contredire entre eux (Descola 1993, p. 137 ; Surrallés 2003, p. 246) : de telles pratiques n'ont de sens que si le rêve est non pas une description d'un état futur auquel il n'est lié que par un lien semblable à celui d'un 
discours à son objet, mais un fait associé à l'état futur par un lien de cause à effet. La différence entre les rêves littéraux et métaphoriques n'est donc pas du même ordre que celle entre langage au sens propre et langage au sens figuré, mais entre action achevée et action amorcée : c'est cet écart entre ébauche et accomplissement que l'« interprétation » doit combler. On pourrait donner pour exemple le fait que, dans les "présages» de chasse, les animaux sont vus comme des humains ; autrement dit, et si l'on en croit Viveiros de Castro (1998), dans une situation de proximité et d'équilibre relatifs : proximité qu'il reste à achever (trouver le gibier) et équilibre qu'il faut renverser à son profit (tuer le gibier, et non être tué, blessé ou séduit par ce dernier) ${ }^{3}$. Évidemment, cela ne s'applique pas de façon aussi simple à chaque fois ; dans certains cas, il semble que le " présage » soit d'assumer provisoirement le point de vue opposé, comme lorsque le succès à la chasse est annoncé par l'impression d'être fléché (Guss 1980). Cette hypothèse peut être étayée par un faisceau d'arguments. Dans les groupes où les rêves métaphoriques ne sont pas mentionnés, les rêves sont en général conçus comme une interaction entre le rêveur et l'interlocuteur "rêvé », d'emblée très forte, aux conséquences favorables ou défavorables, mais toujours immédiates. Chez de nombreux Pano - Cashinahua, Amahuaca, Shipibo -, on peut se battre et être blessé, avoir des relations sexuelles et tomber enceinte ou malade du fait des actions du rêve (Keifenheim 2002 ; Carneiro 1964 ; Leclerc 2003, p. 323). Il en va de même chez les Tapirapé ou les Bororo (Wagley 1977 ; Crocker 1985, p. 204). Quant aux Yanomami, s'il ne semble pas y avoir d'inversions « métaphoriques » dans leur vie onirique, ils acquièrent en revanche des capacités cynégétiques ou guerrières à travers des rêves d'apparence assez « littérale » - sous la forme de l'animal qui en est le paradigme (Albert 1985, p. 156) :l'écart est peut-être signifié par la distance qui va de l'aptitude à l'acte. Par ailleurs, tantôt métaphoriques, tantôt littéraux, les " présages » néfastes sont difficiles à classer : serait-ce parce qu'ils mettent en jeu une relation qui n'est encore qu'en puissance (par exemple la préparation d'une attaque guerrière) ou bien déjà en train de s'actualiser (comme une attaque chamanique ou une maladie) ? Chez les Miraña, certains rêves néfastes sont métaphoriques et prophétiques, tandis que d'autres renvoient littéralement à l'acte de contracter la maladie en se précipitant sur son ennemi embusqué. De plus, les rêves de mauvais présages, même chez les Achuar, peuvent être un acte ou, du moins, la conséquence d'un acte passé, comme une contamination par le spectre vengeur (emesak) de l'ennemi (Karadimas 1997, p. 441 ; Descola 1993, p. 431). Notons enfin que, dans les cas où coexistent interprétations littérales et métaphoriques, les premières concernent des interactions plus fortes et des enjeux plus importants que dans les secondes : obtention d'un esprit protecteur, de chants rituels, etc.

On ne peut donc pas établir de distinction radicale entre des interactions qui seraient des actes réels et d'autres qui ne seraient que des signes à interpréter. L'opposition entre communications «littérales » et celles dites «métaphori- 
ques » peut se réduire à une différence de degré dans l'intensité et l'achèvement de l'interaction entre l'humain et son interlocuteur. Cette intensité et cet achèvement sont fonction de la relation entre ces interlocuteurs, c'est-à-dire de leurs positions respectives, actuelles et à venir, craintes ou désirées par l'un ou par l'autre. La « métaphore " n'est que l'écart entre une position actuelle et celle qu'on infère ou espère pour bientôt, par déduction de la tendance qui se manifeste dans la première. La notion d'interprétation est donc peu satisfaisante pour décrire l'oniromancie amazonienne, dans la mesure où il s'agit moins d'associer un signifiant à un signifié, que de découvrir, dans une situation, les détails permettant de déduire son évolution probable. Il serait donc plus approprié de parler de diagnostic et de pronostic au sens médical, c'est-à-dire d'une hypothèse vraisemblable construite après analyse d'une somme d'indices rassemblés empiriquement. Le choix d'un vocabulaire médical n'est pas arbitraire : le fait de «faire chanter 》 - soit séduire et attirer - un tapir et de découvrir les « causes » d'une maladie - i.e. identifier un agresseur -, ne relève-t-il pas souvent, en Amazonie, d'une même forme de " compétence »? Ce qui explique aussi pourquoi un même élément puisse, selon le contexte, être l'objet de diagnostics très divergents. Pour prendre un trait récurrent, les rêves érotiques peuvent annoncer des issues tout à fait opposées, depuis le succès à la chasse jusqu'à la blessure ou la mort d'origine animale ou humaine : il s'agit d'un moment de séduction pendant lequel les intentionnalités peuvent être perçues à la fois comme égales et rivales; reste à savoir laquelle l'emportera, celle du rêveur ou celle du « rêvé ».

\section{TranSMISSION D'INFORMATIONS ET ACQUISITION DE POSITIONS ÉNONCIATIVES}

Intéressons-nous à présent aux types de «contenus » informationnels qui peuvent être échangés avec les non-humains dans ces relations dialogiques. S'agit-il vraiment de communication au sens d'une transmission d'informations " constatatives »? ou est-ce essentiellement l'aspect performatif qui importe puisque, dans de nombreux cas, ces événements communicationnels constituent un enjeu social, voire vital?

Bien que la seconde hypothèse semble plus probable que la première, dans la mesure où les faits de communication avec des non-humains ne sont pas limités à des conversations triviales ou à des bavardages quotidiens, l'hypothèse d'une transmission d'informations « neutres » ne doit pas être écartée d'emblée puisque c'est sous cette forme que se présentent apparemment certains cas. Le thème de la transmission de nouvelles sur des parents absents ou éloignés, relativement rare en Amazonie, est attesté bien que sur un mode mineur. On le trouve chez les Amahuaca ou chez les Aguaruna pour qui la communication avec les esprits yoshi et pasuk permet de s'informer sur l'état de santé d'individus absents (Carneiro 1964 ; Brown 1985, p. 62). En dépit des apparences, ces informations sont cepen- 
dant loin d'être neutres : l'état de santé des parents, absents ou non, n'est qu'un symptôme des tensions sociales et des rapports de forces « réels » et chamaniques. Cette situation concerne donc très directement l'interrogateur - tout comme les "présages » s'adressent, surtout lorsqu'ils sont néfastes, moins à un individu particulier qu'à un groupe de consanguins et/ou de co-résidents. De même, dans les cas de "présages » et autres rêves annonciateurs, il y a en surface une transmission d'informations, puisque le rêveur apprend que c'est telle ou telle espèce qu'il peut chasser, tel ou tel type d'ennemi qu'il doit redouter - certains rêveurs sont même parfois spécialisés dans un domaine particulier d'informations, tels ces Tapirapé qui, ayant acquis comme auxiliaires des esprits de Kayapo morts, peuvent annoncer les attaques de Kayapo vivants (Wagley 1977). Cependant, l'information transmise et le contenu du dialogue n'excèdent jamais le fait même de la communication et ses modalités formelles : tout son sens ou, plutôt, tous ses sens possibles se limitent à la relation avec un interlocuteur et aux modalités de manifestation de ce dernier - à travers une apparence anthropomorphe ou zoomorphe, masculine ou féminine, prédatrice ou séductrice, etc. -, puisque tout cela ne fait qu'exprimer l'état tendanciel de la relation entre les intentionnalités respectives des deux interlocuteurs. Hormis cela, il n'y a guère d'information pertinente. À la limite, les cas apparemment les plus informatifs sont peut-être les plus clairement performatifs, à la fois parce que la nature de l'interlocuteur est prédéterminée par la spécialisation communicationnelle du sujet humain et parce que l'information y est particulièrement précise et riche, portant sur des données autres que les dispositions relationnelles de cet interlocuteur, comme des lieux ou des dates. On peut donner pour exemple ces chamanes juruna anciennement experts en prise de rendez-vous avec les pécaris, annonçant le jour et le bras de rivière où les chasseurs pourraient les rencontrer pour une " fête » (Lima 1996) : ce ne sont pas tant les pécaris qui notifient leur venue au chamane que celui-ci qui les séduit (très explicitement, en jouant de la «musique » porcine) et qui estime ensuite le degré d'eflicacité de son charme, estimation exprimée en distances spatiale et temporelle.

De plus, lorsque le dialogue avec un non-humain est l'occasion de la transmission d'une information ou même d'un " savoir », ce savoir présente avant tout une valeur différentielle par rapport au savoir humain, c'est-à-dire à celui des autres humains : il s'agit de faire reconnaître par ces derniers, selon diverses modalités de validation, ce fait de communication, son degré de crédibilité et, éventuellement, d'intimité, de distance ou de nouveauté. Le contenu informatif n'y est donc qu'une caractéristique secondaire, preuve éventuelle de la communication et mesure possible de son intimité et de sa force. Cette conception de l'information par et sur les non-humains, comme mesure d'un degré de puissance, se retrouve d'un bout à l'autre des Basses Terres : depuis le chamanisme des divers Tukano jusque dans les rapports des Bororo aux esprits bope, en passant par les groupes du Haut Xingu ou par certains Pano comme les Shipibo ou encore par 
les Yagua - puissance qui peut, selon les cas, s'exprimer dans divers domaines, depuis la chasse jusqu'à la médecine. Il serait difficile de citer une interaction dont l'aspect performatif n'apparaisse pas au moins aussi important, sinon plus, que son contenu informatif - et ce dernier aspect est bien évidemment encore plus négligeable lors des interactions involontaires de séduction par un esprit. Même dans les cas où une information apparemment " utile » se surajoute à un aspect performatif qui se suffit à lui-même - comme dans certaines révélations d'arutam chez les Candoshi où l'apparition « révèle le déroulement d'un prochain raid » (Surrallés 2003, p. 186) -, il semble que cet aspect informatif soit tenu pour relativement négligeable en tant que tel : tantôt ce n'est qu'une modalité autre d'attestation de réussite de la quête, tantôt il se surajoute à d'autres, mais il ne semble pas que le sujet en tienne réellement compte dans la tactique de ses guerres ultérieures - au-delà, bien sûr, de la " prédiction » sur leurs issues, heureuses ou funestes.

Faudrait-il alors ne prendre en compte, dans toutes ces formes de communication, que leur aspect performatif en général qui serait seul signifiant, pour réduire les communications avec des non-humains à une "métacommunication » batesonienne, comme le propose Bird-David (1999) pour des faits semblables? Dans cette perspective, toute communication non humaine consisterait uniquement à «communiquer qu'on communique ». Une telle réduction ne peut être que spécieuse, tant qu'on n'a pas au moins précisé à qui " on communique qu'on communique ». L'hypothèse qui en découle est celle de la stratégie sociale. La maîtrise des relations avec les non-humains et l'appropriation symbolique de leurs pouvoirs serviraient à acquérir; sinon un pouvoir; du moins un prestige social. Peu pertinente dans les cas d'interactions néfastes et involontaires, cette explication sociologique n'est cependant pas sans arguments. On connaît de nombreux cas où le statut de chamane, voire le simple fait de savoir raconter une interaction passagère avec des non-humains, est un facteur de prestige, prestige qui réside parfois en un pouvoir « objectivé »-par un chant, un charme ou des " fléchettes » invisibles -, réifié et transmissible contre paiement. Ce prestige fournit souvent le langage des luttes politiques entre factions dont la puissance est notamment quantifiée d'après le nombre d'interlocuteurs spirituels que ces factions sont supputées maîtriser. Il intervient directement dans ces luttes politiques, comme la maladie ou la mort provoquée par la manipulation d'esprits malveillants est un motif majeur de guerre, voire une arme politique dissuasive, par exemple chez les Yanomami, dans les groupes du Haut Xingu ou chez les Jivaro : la puissance politique ne peut être dissociée de capacités chamaniques reconnues, dans la personne même du grand homme ou dans celle d'un de ses alliés (Albert 1985 ; Gregor 1977 ; Descola 1993). Car la maîtrise de la communication avec les non-humains, en tant qu'elle procure à la parole l'autorité d'une origine extérieure et/ou d'un langage supposé désintentionnalisé, peut permettre de légitimer un discours portant sur les relations et tensions inter-humaines : le 
chamane araweté ne se prive pas d'attribuer aux dieux et aux morts des remarques sur des affaires matrimoniales en cours (Viveiros de Castro 1992, p. 234 ; Taylor 1993a).

Cependant, cette maîtrise n'est pas toujours conçue comme socialement bénéfique à son détenteur, ni même parfois à ses proches parents. Dans certains groupes pano, le chamanisme n'est qu'un souvenir, nul ne souhaite se lancer dans cette carrière potentiellement utile d'un point de vue collectif, mais très défavorable d'un point de vue individuel ou familial. Le chamane cashinahua était perçu comme l'antithèse du chasseur et du chef, certes capable de tout entendre, mais incapable de parler efficacement, privé de viande et de femme ; en conséquence, une vocation chamanique était vécue comme une malédiction par le groupe familial (Deshayes 1992; Kensinger 1995). Aujourd'hui, chez les Katukina, même l'acceptation de la carrière de "détenteur de chants ", forme faible du chamanisme disparu, ne peut se faire sans une discussion et un accord des parents proches (Coffaci de Lima 2000, p. 145). On retrouve une telle ambivalence dans le statut de communicant privilégié chez le chamane bari bororo : certes, il est payé pour sa façon de traiter la nourriture, mais tous le considèrent avec défiance sinon détestation (Crocker 1985). L'explication sociologique peut bien sembler pertinente quand cette maîtrise résulte d'une quête consciente et volontaire - et $a$ fortiori quand il faut acheter ce savoir auprès d'un prédécesseur. Mais cette hypothèse rend difficilement compte des cas où la communication avec un non-humain est avant tout l'effet d'une « élection » par les esprits. Enfin la théorie deviendrait franchement absurde lorsque l'interaction est involontaire et néfaste. Sous cette forme quelque peu grossière, la notion de communication performative ne mène qu'à des tautologies : dans les cas où un pouvoir est conçu comme étant accessible uniquement au moyen de la communication non humaine, le fait, la réussite et la preuve de ladite communication donnent une reconnaissance sociale de ce pouvoir et parfois sa réalité.

Sans abandonner l'idée de la primauté des aspects performatifs dans ces formes de communication, il semble nécessaire d'en faire une analyse plus fine en prenant en compte la diversité des contenus concrets et apparents de ces dialogues pour tenter de les corréler à différents types d'effets performatifs, en considérant aussi bien des résultats bénéfiques que des conséquences défavorables. Pour cela, on s'appuiera sur l'hypothèse de Viveiros de Castro (1998) qui définit, au sein des " déictiques cosmologiques », la position de « surnature », c'est-à-dire des nonhumains communicants, comme « seconde personne » grammaticale. Or on sait qu'en Amazonie on s'adresse rarement à quelqu'un par un simple «tu », on utilise plutôt un terme de parenté pour préciser et qualifier la relation qu'on entretient ou qu'on veut entretenir avec l'interlocuteur. On peut donc émettre l'hypothèse qu'il en va de même avec les non-humains et qu'il est souvent possible de déterminer, en fonction des termes de parenté utilisés par les humains dans leurs dialogues avec les non-humains, les diverses relations que les uns cherchent 
à entretenir, simuler ou éviter avec les autres. Cela dit, toutes les interactions ne pourront être prises en compte dans ce modèle, car l'utilisation des termes de parenté n'est pas toujours précisée - elle est même très improbable - dans les interactions non linguistiques ${ }^{4}$. Toutefois les communications non linguistiques sont souvent des interactions ou bien très faibles - anodines, avortées et inefficaces -, ou bien extrêmement fortes et rapidement "achevées 》-i.e. exhibant immédiatement leur « sens » performatif, en général la mort d'un des interlocuteurs. Enfin, lorsque ces termes de parenté sont absents ou inconnus de nous, on peut tenter de reconstruire et d'identifier les relations de parenté à partir d'indices (comme les émotions, les objets échangés, le style rhétorique, etc.) constitutifs du modèle sur lequel ces interactions sont pensées.

\section{VALEURS RELATIVES DES DIFFÉRENTES POSITIONS}

En Amazonie, un des principes de parenté présentant la plus forte récurrence et un grand rendement symbolique dans la conception des relations avec les différentes formes d'altérité humaine et non humaine est l'opposition fondamentale des systèmes dravidiens entre consanguinité et affinité (Descola 1994). Cependant, tout en prenant comme point de départ cette distinction, nous avons pu mettre au jour plusieurs figures de l'affinité et, plus encore, de la consanguinité dans une trentaine de cas, provenant d'une quinzaine de groupes différents, où nous avons réussi à identifier des relations de parenté. Ces configurations sont plus ou moins étroitement en corrélation avec d'autres aspects caractérisant les interactions en question.

La présence de relations d'affinité avec les non-humains n'est pas surprenante dans la mesure où l'affinité, au sens d'« affinité potentielle » ou « méta-affinité », est la forme générique et « non marquée » de l'altérité en Amazonie (Viveiros de Castro 2001 ; Taylor 2000) : par définition, des relations avec des non-humains sont ontologiquement et sociologiquement distantes. Or le méta-affin, sinon l'affin réel, est aussi la figure première de l'ennemi. Dans ces relations d'affinité, on trouve donc de nombreux cas - par exemple chez les Jivaro, les Tukano, les Yagua, les Juruna ou encore les Tapirapé - concernant la chasse et, plus particulièrement, la séduction de la proie. Il s'agit bien d'une relation empreinte d'incertitude et de violence, finissant en meurtre. De fait, dans les cas de relations affines non cynégétiques ou non directement cynégétiques, il semble qu'on puisse retrouver ces caractéristiques. C'est alors en général une acquisition d'auxiliaires chamaniques. Celle-ci est, au moins par comparaison avec d'autres acquisitions, tantôt instable et susceptible de dissolution - chez les Achuar par exemple (Descola 1993, p. 357) -, tantôt franchement conçue comme prédatrice et dangereuse - chez les Tapirapé (Wagley 1977, p. 203). Dans le chamanisme wari', elle résulte d'une chasse de l'humain par l'animal, laquelle est perçue en continuité 
avec certaines maladies et dont l'issue est une capture définitive du chamane par les animaux entraînant sa mort (Vilaça 1999). Enfin une telle relation d'affinité apparaît aussi entre l'artisan warao et l'arbre dont il doit faire une pirogue, activité très importante et, semble-t-il, marquée par l'incertitude (Wilbert 1993, p. 69).

Toutefois ces relations d'affinité ne sont pas toutes identiques et les nonhumains peuvent se voir attribuer des positions variées. Ces relations impliquent d'emblée une dualité chez les non-humains qui occupent toujours deux positions. Il s'agit là d'un trait cohérent avec la parenté humaine, puisqu'il reproduit la double nature constitutive des affins humains avec, d'un côté, les femmes que l'on doit s'approprier et, de l'autre, les hommes qu'il faut éviter ou tuer (Taylor 2000). Cette dualité laisse apparaître l'impossibilité d'une socialisation - sous forme de consanguinisation - complète et d'un contrôle absolu sur les non-humains ${ }^{5}$. Cependant ce dédoublement des positions peut être plus ou moins marqué : on a tantôt une distinction de génération et de sexe (Desana), tantôt seulement l'une (Jivaro) ou l'autre (Makuna) ou, encore, ni l'une, ni l'autre, la distinction se résumant à une simple différence de statut (Juruna). Ces variations ne semblent pas insignifiantes. Lorsque la dualité est faiblement marquée, comme chez les Juruna entre les pécaris et leur "chamane", le pôle que l'on peut qualifier de féminin est relativement plus dangereux, le pôle masculin relativement moins puissant, moins hors de portée des humains : les pécaris peuvent voler des âmes humaines durant la chasse collective, mais leur "chamane », animal réel et morphologiquement semblable aux autres, risque, bien que ce soit une bourde à éviter, d'y être tué (Lima 1996), mésaventure qui n'arrive jamais, dans la plupart des autres groupes, à la figure classique du Maître des animaux, entité rarement rencontrée lors d'une chasse. L'autre divergence notable apparaît entre les dualités marquées par une différence de sexe et celles qui le sont par une différence de génération. En effet les premières semblent être en corrélation avec des relations fondées sur une réciprocité acceptée, bon gré mal gré, par les humains. Par exemple, dans les relations cynégétiques chez les Miraña, les Makuna ou les Desana, la proie est conçue comme féminine et épouse ; il faut donc offirir à son " père », « frère » ou « maître », divers « biens », « nourriture » (coca, tabac, etc.) ou « paiements » (en « âmes » humaines). Cette réciprocité engendre parfois la tentation d'escroquer son " beau-père » en substituant des images érotiques au don de ses sœurs humaines (Karadimas 1997, p. 374 ; Arhem 1996; ReichelDolmatoff 1973, pp. 109, 160). Dans cette catégorie, mentionnons une forme apparemment inversée dans sa formulation, celle des rituels yagua, visant à placer les esprits de la chasse en position de preneurs de femmes humaines : celles-ci jouent un rôle d'épouse en leur offrant de la bière ; eux, tout comme des gendres, devront fournir de la viande à leurs affins (Chaumeil 2000, p. 187). Cette réciprocité inversée, où l'humain garde la position dominante de beau-père, est peut-être une forme intermédiaire entre la prédation pure, à laquelle semblent tendre habituellement les Yagua, et la négociation régulièrement consentie par les 
Desana, en position de gendres face au Maître du gibier : les Yagua ne s'y résolvent qu'en cas de disette. La réciprocité se rencontre encore, de manière beaucoup plus floue, dans les acquisitions d'auxiliaires chamaniques affins, lorsque la dualité inclut une différence de sexe. C'est le cas des « filles de Tsunki » chez les Achuar : cette relation conjugale idéale implique, à tout le moins, que l'humain s'abstienne d'agresser le père de la charmante auxiliaire, voire qu'il lui " donne » une de ses filles (Descola 1993,p. 163). Notons par ailleurs que la dualité inverse, où le non-humain non approprié serait féminin et le non-humain approprié masculin, n'existe pas : il s'agirait d'une contradiction dans les termes. Au contraire, lorsque la dualité n'est marquée que par une différence de génération, la relation prend la forme d'une prédation avouée, sans réciprocité. C'est le cas dans la chasse jivaro où la proie est conçue comme un « beau-frère ", ennemi à tuer, du même sexe que son " père ", le Maître des animaux - et ce n'est que durant le temps nécessaire à sa séduction (la préparation à la chasse et la chasse elle-même) que cette proie est hypocritement féminisée. De même, pour acquérir des auxiliaires, les Tapirapé les affrontent violemment ; ces auxiliaires se présentent sous la forme du « fils » d'une entité masculine. En revanche, la chasse comporte pour les Tapirapé une forme de réciprocité, grâce à une différence sexuée : le chamane doit se résoudre à " féconder » les pécaris femelles pour pouvoir chasser les mâles (Descola 1992 ; Taylor 2000 ; Wagley 1977). Si, dans les exemples précédents, la masculinité du non-humain est toujours associée à une relation agonistique, il semble néanmoins que l'absence de différence sexuée entre les deux pôles du non-humain suffise à marquer la non-réciprocité et, par conséquent, la tendance à la prédation : dans l'abattage de l'arbre à pirogue chez les Warao, opération majeure et délicate, l'arbre est conçu comme un être féminin dépendant d'une « mère » qui ne reçoit rien en échange de la séduction de sa fille (Wilbert 1993). L'affinité comporte donc plusieurs figures à partir d'une forme propre, celle de la dualité, qui peut être marquée de diverses manières et recevoir différentes configurations, toutes n'étant pas possibles et n'ayant pas la même valeur.

Le cas des relations de consanguinité est plus complexe. Elles sont en effet plus riches puisqu'elles peuvent prendre des formes variées. On peut distinguer quatre types, plus ou moins récurrents, de configuration principale attribuant au nonhumain les positions de grand-père, fils, mère et père. Trois propositions peuvent être avancées concernant ces relations décrites en terme de consanguinité. D'abord, comme dans les relations affines, une dualité y est présente, mais virtuellement : bien qu'expressément consanguines, ces positions sont inséparables de relations d'affinité préalables, simultanées ou éventuelles. Ensuite, les relations avec un ascendant s'opposent à celles avec un descendant, les premières étant beaucoup moins contrôlées, ce qui n'est pas pour surprendre. Enfin, ce qui aurait pu paraître moins évident, les deux positions à $\mathrm{G}+2$ et $\mathrm{G}-1$ s'opposent à celles à $\mathrm{G}+1$, semble-t-il, comme nettement plus favorables dans la mesure où les secondes sont très ambivalentes, sinon franchement négatives. 
Les positions en $\mathrm{G}+2$ se résument à celle de « grand-père » car on n'a trouvé aucun cas "féminin » dans cette position. Par extension, nous assimilons les positions décrites comme celle d' " ancêtres », notamment chez les Xavante ou les Mundurucu, c'est-à-dire logiquement des gens avec qui on entretient un rapport de filiation lointaine ${ }^{6}$. Cette position de " grand-père » est en effet étroitement en corrélation avec trois caractéristiques de ces interactions. D'une part, il s'agit d'une relation positive, de protection du sujet humain par le non-humain. C'est le cas des spectres arutam jivaro, de certains auxiliaires mehinaku ; c'est expressément le type de relation que les Desana veulent établir avec un jaguar réel lorsqu'ils passent à proximité d'un de ses repaires et s'adressent à lui afin que celui-ci ne les attaque pas (Taylor 1993a; Gregor 1977, p. 328 ; ReichelDolmatoff 1973, p. 103). Bien que moins marquée, cette caractéristique se conserve dans les positions d' " ancêtres » : l'interaction est bénéfique ou, tout au moins, relativement peu périlleuse, en tout cas pour les hommes adultes - ou initiés -, si on y inclut des trompes et tubes sacrés, considérés comme ayant une forte connexion avec l'ancestralité chez les Yagua et comme dangereux pour les femmes. D'autre part, il s'agit d'une relation dans laquelle des pouvoirs sont transmis et objectivés sous une forme plus ou moins publique : les Xavante, les Mundurucu et les Mehinaku obtiennent, de leurs " ancêtres » ou "grandspères » rencontrés en rêve, des chants et des danses qu'ils représentent publiquement ensuite (Graham 1995; Murphy 1958 ; Gregor 1977). Quant aux arutam jivaro, ils restent secrets, tout en constituant une transmission de puissance objectivée par un comportement assez reconnaissable (Surrallés 2003, p. 189). Enfin, ces relations sont souvent pensées comme voilant - ou ayant d'abord été voilées par - une relation d'affinité, que la position de " grand-père » doit nier. Le cas le plus clair est celui des Mehinaku. C'est d'abord en tentant de tuer, donc dans une relation de méta-affïnité extrême, un cervidé qui se trouve être la "femme » d'un esprit, que le sujet humain entre en contact avec ce " grandpère », lequel a une apparence d'étranger sauvage (non xinguano); cette position finale de "grand-père » doit interdire l'actualisation de la relation de métaaffinité antérieure. Dans le cas jivaro, la position et l'aspect de " grand-père » ne sont que l'apparence d'arutam et, surtout, on commence par s'adresser à lui par des chants anent, lesquels ne sont normalement jamais chantés pour des consanguins (Taylor 2000). Chez les Mundurucu, ce fond d'affinité est moins visible. On peut cependant noter que, comme chez les Xavante d'ailleurs, cette relation s'établit au moyen du rêve, autrement dit d'un "voyage de l'âme ", activité désocialisée de l'organe affïn de l'humain amazonien (Viveiros de Castro 2001). Enfin les différents villages xavante rivalisent pour obtenir le plus grand nombre de chants de leurs « immortels » afin d'attester leur proximité relative plus étroite avec ceux-ci (Graham 1995, p. 170) : on peut y voir une compétition entre affins pour affirmer un degré supérieur de consanguinité avec les « immortels ». Cette hypothèse d'une compétition entre affins humains potentiels pour l'affirmation 
d'une ascendance non humaine pourrait d'ailleurs être étendue à d'autres cas, notamment peut-être aux quêtes d'arutam jivaro ou d'auxiliaires chamaniques et aux rituels chez les Mehinaku ${ }^{7}$. On trouve une forme inversée de cette configuration dans la relation que les Waiwai «baptisés » tentent d'établir avec les « tribus inconnues » (non évangélisées) : les premiers essaient de se faire reconnaître par les seconds comme des ancêtres mythiques et des héros civilisateurs ; ils deviennent les protecteurs et les donneurs de biens culturels (chrétiens) de ces anciens méta-affins et ennemis (Howard 2001, p. 408).

Les positions à $\mathrm{G}-1$ possèdent deux formes majeures : il peut s'agir d'un fils masculin non humain, "de nature spirituelle », ou bien d'un enfant " réel », masculin ou féminin. Sans surprise, la seconde forme est toujours le produit de l'union d'une humaine et d'un non-humain, tandis que la première concerne essentiellement des sujets humains masculins. Il n'est guère plus surprenant que la première position exprime une forme de contrôle du père humain sur son fils non humain. Chez les Achuar, les Yagua, les Huaorani, les Waiwai, les Tapirapé ou les Kaingang, la plupart de ces « fils » sont en effet des auxiliaires chamaniques. Lorsque, comme chez les Achuar, ces auxiliaires filiaux cohabitent avec des auxiliaires affins, les premiers sont beaucoup mieux contrôlés par le chamane (Descola 1993, p. 357). On peut aussi trouver dans ce cas un rapport d'affinité préalable ou éventuelle. Préalable, car tantôt le « fils » non humain a d'abord dû être séduit pour être acquis comme auxiliaire - voire être re-séduit à chaque emploi, par exemple chez les Yagua qui « ingèrent » leur « fils » durant l'initiation et dont les chants chamaniques, dans leur partie initiale, ont une connotation d'« invitation sexuelle »-, tantôt cette consanguinisation ne s'achève même pas : les auxiliaires des Mastiguenga ne sont que leurs « gendres » (Baer 1992). Cette position d'aftin en $\mathrm{G}-1$, co-résident, " consanguinisable » au service de son beau-père, constitue sans doute un intermédiaire entre les positions attribuant aux auxiliaires chamaniques un statut de beaux-frères ou de beaux-pères et celles leur attribuant un statut de fils, plus sûr que des alliés en $G 0$ ou $G+1$, mais moins que des fils. Tantôt enfin le « fils » a plutôt la forme d'un captif, adopté et "consanguinisé », puisqu'il doit être acquis dans une relation agonistique, comme dans l'initiation chamanique tapirapé (Chaumeil 2000, p. 115 ; Wagley 1977 , pp. 184, 203). Cette relation d'adoption, certes moins pensée de manière agonistique que comme une forme d'alliance alternative au mariage, se retrouve chez les chamanes huaorani dont les auxiliaires sont des « fils de jaguar » ou chez les Kaingang dont les auxiliaires sont des "enfants d'esprits » adoptés (Rival 1996 ; Henry 1964, p. 73). Mais, même acquise, l'affinité se maintient comme un risque éventuel : le « fils » reste toujours susceptible d'être « séduit » par un autre chamane, le vol et la perte d'auxiliaire étant un thème classique du chamanisme amazonien. Il est cependant difficile de distinguer ce qui relève de la séduction exercée directement sur l'esprit - placé en position féminine ou, à la limite, en position de fils quittant ses parents pour un beau-père selon la règle d'uxoriloca- 
lité - de ce qui relève du combat chamanique et de la prise de captifs - laissant l'esprit en position d'enfant asexué. Les deux sont souvent présents : par exemple, chez les Yagua, un chamane peut s'y faire voler un auxiliaire si un rival découvre le « chant » auquel il répond ou bien les lui abandonner tous s'il succombe lors d'un duel chamanique (Chaumeil 2000, p. 118). Le sexe, bien que toujours masculin, ne paraît donc pas être un caractère très important dans cette position. Cette affinité éventuelle n'est jamais qu'un résultat logique de la position d'enfant, puisqu'un enfant est toujours un moyen d'acquérir des alliés, donc d'entrer dans une relation d'affinité. Cette position est d'ailleurs instable : chez les Kaingang, il semble qu'elle ait été remplacée par une simple relation d'aftinité, marquée par la " vive jalousie » de l'esprit à l'encontre de l'épouse humaine du chamane (Crépeau 2000). Toutefois, si cette position d'enfant dénote une relation à un non-humain plus stable que celle d'affinité et si, comme celle-ci, elle fournit des pouvoirs, ceux-ci ne sont pas de même degré, ni de même nature. Revers de la stabilité, les pouvoirs conférés par les esprits filiaux sont souvent bien inférieurs à ceux des esprits affins. Cela se voit dans les cas où les deux types coexistent, comme chez les Achuar et les Yagua dont les auxiliaires sont bien inférieurs aux esprits de la chasse, plus dangereux : cas particulier du principe général selon lequel plus on est lointain, plus on est puissant (Descola 1993, p. 374). Il est en revanche plus ardu de comparer les positions de « grand-père » et de « fils » du point de vue de la stabilité et du degré de puissance. Toutefois on peut, semble-t-il, y voir une opposition entre, d'un côté, une interaction brève, relativement indépendante de la volonté du sujet, unique et/ou impossible à reproduire comme événement (telle quête précise, tel rêve) conférant un pouvoir durable, constant et général comme le " blindage spirituel » d'arutam ou la maîtrise d'un chant et, de l'autre, des interactions répétées et fréquentes (séances chamaniques) à partir d'une communication initiale fournissant des savoirs ou des pouvoirs utiles à court terme pour des problèmes relativement précis (une cure, un besoin momentané de gibier; etc.). Les Kaingang tendraient certes à affirmer le contraire puisque l'esprit adopté « suit partout » son père humain ; cependant chaque type d'esprit ne fournit qu'un pouvoir limité à tel ou tel gibier (Henry 1964). Quant aux Yekuana, s'ils décrivent le chamanisme comme une relation avec un ascendant à $\mathrm{G}+2$, c'est en insistant sur le fait que, son âme (akato, "double») se transformant définitivement en " grand-père esprit » (sadashe), l'être du chamane devient intrinsèquement et continûment différent de celui des humains ordinaires (Guss 1980).

La seconde forme de position à $\mathrm{G}-1$ est en apparence bien différente, puisqu'il s'agit d'enfants « réels ». Ces croyances attribuant à certains enfants un père non humain se rencontrent dans de nombreux groupes: Shipibo, Amahuaca, Kaingang, Mundurucu, Palikur, Parintintin (Saladin d'Anglure et Morin 1998 ; Carneiro 1964 ; Henry 1964 ; Murphy 1958 ; Passes 1998 ; Kracke 1990) ${ }^{8}$. Néanmoins l'attraction de cette position vers l'affinité non humaine reste alors 
visible. Si, par définition, elle est toujours initialement présente, il semble que, dans de nombreux cas, elle s'y maintienne, ces enfants étant impossibles à socialiser, "consanguiniser » et humaniser entièrement. Leur corps est souvent laid et difforme (Palikur, Waiwai), sinon handicapé (Shipibo, Amahuaca). Ils sont maladifs (Mundurucu), en rapport étroit avec leur espèce paternelle (Kaingang), ou tout simplement chamanes (Parintintin), autrement dit attirés par le lointain et l'aftinité. Ces positions peuvent donc être tantôt bénéfiques pour le groupe, sinon pour ces enfants, tantôt dangereuses pour eux-mêmes et pour les parents.

Au contraire des positions précédentes, dénotant des relations positives, les positions à $\mathrm{G}+1$, lorsqu'elles sont attribuées au non-humain, se révèlent très ambivalentes, voire franchement négatives. Il semble rare que ces positions soient données explicitement et directement à un interlocuteur non humain. Nous n'en connaissons qu'à peine trois cas où le non-humain se voit systématiquement accorder un sexe masculin et est toujours placé dans une relation, sinon d'hostilité déclarée, du moins de tension ${ }^{9}$. Ainsi, chez les Parakanã, les interlocuteurs rêvés grâce auxquels on obtient des chants sont considérés comme des " autres », des non-parents, des « ennemis » (akwawa), qu'il s'agit de transformer en animaux familiers - c'est-à-dire de placer en position filiale. Les termes de référence dénotent cette ambiguïté : ils sont à la fois décrits comme des " animaux familiers », comme des « proies » ou " victimes » et comme des « jaguars ». Mais, au cours de l'interaction en rêve, donc de la transformation de ces interlocuteurs ennemis en enfants soumis, le rêveur s'adresse à eux comme à des "pères" (miangá, $\mathrm{F}$ ou $\mathrm{FB}$ ). Ce vocatif était aussi celui utilisé pour parler aux Blancs lors de la " pacification ». Certes, comme le souligne Fausto, on ne saurait voir dans cette position uniquement un aspect négatif : ces " pères » cèdent leurs pouvoirs aux rêveurs parakanã et les protègent donc - certains sont d'ailleurs parfois nommés " grands-pères ». Néanmoins, ce terme d'adresse semble attaché à un moment d'incertitude - le rêve et la pacification comme première " rencontre " avec l'ennemi onirique ou lointain - et ces " pères » ne sont pas de véritables consanguins. En effet, les plus puissants - i.e. les plus prédateurs - sont précisément nommés « oncles maternels », position nettement plus affine (Fausto 1999, p. $939 ; 2001$, pp. 348, 472). On retrouve chez les Bororo une même ambivalence dans la relation tendue entre le chamane bari et ses « pères » bope. Si certains de ces esprits le protègent, ils le font aussi souffrir, l'affligent fréquemment de maladies et le considèrent, lui, son épouse et ses biens, comme leur propriété dont ils peuvent disposer à leur guise. Là encore, la position de père est marquée par un rapport complexe à l'affinité. Certes le bari est de la moitié alterne (Tugarege) à celle de ses « pères » bope (qui relèvent de la moitié Exerae), en conformité avec l'exogamie matrilinéaire des Bororo. Mais les esprits bope étant toujours irrespectueux des règles sociales et incestueuses, la pratique du chamanisme implique qu'on leur laisse profiter de leur épouse, nécessairement Exerae. Ces " pères » 
nominaux se conduisent en réalité comme des affins, ou plutôt comme des méta-affins, qui conservent incestueusement leurs « sœurs cadettes »-terme par lequel les bope sont censés désigner les femmes Exerae (Crocker 1985, p. 206). Enfin la position de père apparaît comme l'un des auxiliaires des chamanes waiwai : s'il ne semble pas y avoir d'hostilité explicite dans cette relation, cet esprit, "Soleil », fait néanmoins partie d'une classe d'êtres (kakenaukworokjam) identifiée par Fock (1963, p. 24) comme particulièrement dangereuse ; de plus, à la différence des autres auxiliaires nommés « fils », les chamanes lui doivent des services - ce qui le placerait plutôt dans la position de beau-père.

Si les positions en $\mathrm{G}+1$ apparaissent comme celles qui sont les plus marquées par l'hostilité, et donc paradoxalement par l'affinité, c'est peut-être parce qu'elles sont avant tout une inversion de celles en $\mathrm{G}-1$ au sens où, au lieu d'exprimer le contrôle du sujet humain sur un auxiliaire non humain, elles dénotent une reconnaissance, momentanée ou définitive, stratégique ou fataliste, d'une soumission à ce non-humain - à l'égal de celles que les animaux entretiennent avec la figure classique de leur Maître, entité souvent dénommée « Père du gibier ». On peut le voir indirectement dans certaines maladies (kep xirak) chez les Wari' où un animal capture un humain pour en faire son « fils » (Vilaça 2002, p. 357) : au lieu que la «consanguinisation » de la relation se réalise au profit du sujet humain, elle se fait à ses dépens - la domination correspondant à la différence de génération. Toutefois, de la part de l'humain, le fait de reconnaître cette sujétion par un terme d'adresse explicite et, qui plus est, sexué - puisque masculin pourrait être compris comme un moyen de maintenir une dose d'hostilité et d'affinité dans cette relation. En effet, cette valeur surprenante de la « paternité » non humaine s'éclaire sans doute par rapport à une autre forme de position en $\mathrm{G}+1$, non sexuée du moins explicitement, mais fortement connotée par la féminité - on peut donc, par commodité, la désigner comme celle de "mère ». Cette position est implicitement présente comme achèvement de certaines interactions négatives. Si ces dernières commencent en général sous forme de séduction, donc dans une relation d'affinité, elles semblent s'achever, au moins dans certains cas, non pas par une alliance matrimoniale, mais par une position de captif, c'est-à-dire d'enfant adopté - comme le suggèrent les maladies wari' -, position dont le paroxysme est l'animal familier. De fait, il existe des nonhumains qui cherchent à se fournir en animaux de compagnie en capturant des humains. C'est du moins ce que viseraient les démons iwianch achuar et certains esprits forestiers desana lorsqu'ils cherchent à séduire un humain (Taylor 1993b ; Reichel-Dolmatoff 1975, p. 194). Or l'apprivoisement - i.e. la familiarisation, la « consanguinisation »-des animaux capturés est pensé en Amazonie comme un rapport maternel, voire est une tâche confiée effectivement aux femmes; et la forme maximale ou paradigmatique de la consanguinité, purifiée de tout rapport d'affinité et donc pacifiée, est illustrée par la relation avec la mère (Taylor 1996; 2000). De plus, la «trajectoire cosmologique » de l'animal domestique amazo- 
nien est celle d'une affinité très lointaine ontologiquement et sociologiquement - c'est un fils d'ennemi non humain - transformée en consanguinité absolue, puisque stérile dans la mesure où on ne laisse pas se reproduire les animaux domestiques. Or, si le processus de séduction d'un humain par un non-humain ne débouche pas explicitement sur une relation du type « animal de compagnie infantilisé - nourrice féminisée », il produit néanmoins des résultats très similaires. Car, sauf quelque cas (chez les Shipibo, les Chimane, ou les Desana), où certains rapports d'affinité avec des esprits sont « fertiles », ces relations aboutissent généralement à une stérilité absolue - notamment imprégnée par l'absence de devenir et par la pure répétitivité (Taylor 1993a ; Surrallés 2003, p. 116). La position de " mère »-implicitement assignée aux non-humains en raison de celle d'animal familier dans laquelle se trouve enfermé le sujet humain - correspond donc à l'aboutissement et à l'échec d'un rapport d'affinité : l'interaction s'achevant dans une domination et un apprivoisement si radicaux du sujet humain, déshumanisé et presque entièrement « désintentionnalisé », que, par contrecoup, son séducteur s'en trouve réduit à une position excessivement féminine et maternelle, donc stérile, comme celle de maîtresse d'animal de compagnie. C'est peut-être en cela que les esprits séducteurs sont plus dangereux que les ennemis humains : ces derniers, en volant des femmes, produisent au moins d'autres humains que l'on pourra tuer et/ou voler en retour; mais, avec les premiers, la perte est définitive.

Les positions en $G+1$, explicites et masculinisées ou implicites et probablement féminines, semblent donc toujours négatives. La distinction de ces deux formes tient sans doute à l'attitude du sujet humain face au non-humain en position dominante. Soit l'humain abandonne toute résistance et s'instaure une relation non conflictuelle, c'est-à-dire une relation dé-masculinisée, au point d'en désexualiser son terme dominé (l'humain transformé en animal familier) et d'en féminiser son terme dominant (le non-humain devenu sa "mère » et "mâ̂tresse »). Dans ce cas, c'est presque une non-relation, puisqu'elle ne peut même plus être formulée directement et explicitement, le point de vue de l'humain se trouvant absorbé par celui du non-humain. Soit l'humain réussit à conserver la conscience de relation de domination et donc de la tension qui lui est inhérente : dans ce cas, cette relation pourra être énoncée et formulée par un terme d'adresse, et ce terme sera masculin. L'humain reconnaît certes alors sa soumission, par la différence de génération, mais il refuse d'abdiquer toute conscience et toute hostilité potentielle en se maintenant dans le cadre d'une relation masculine. Les rêveurs parakanã, experts dans le contrôle rituel des degrés de familiarisation et d'affininité prédatrice de leurs interlocuteurs (Fausto 1999), illustreraient peut-être bien cette situation. Elle pourrait en effet dans ce cas être comprise comme un moment d'une stratégie de familiarisation qui, en s'adressant à l'ennemi rêvé comme à un " père ", viserait à attirer ce méta-affin dans une relation de consanguinité orientée en apparence à son profit. Mais, ce dernier n'ayant pas été l'auteur - ou le maître - de son énonciation et de sa formulation, il sera facile à 
l'énonciateur feignant d'être son fils de renverser cette relation par la suite, dès que ce " père » trop assuré aura commis l'imprudence de lui révéler ses chants ; ce " père " provisoire et paradoxal finira alors en " animal domestique » du rêveur qui, maître de l'énonciation de ses chants, contrôlera désormais les pouvoirs de celui qui s'était cru son " père » et " maître ».

Il n'en reste pas moins que toutes les positions de consanguinité, en corrélation avec des caractéristiques assez bien déterminées des modes de communications, sont en fait différentes formes possibles de "consanguinisation » d'un rapport de méta-aflīnité préalable et, en fonction des formes, dénotées par la combinaison de divers traits contrastifs - ascendance ou descendance, différence ou identité sexuelle, etc. -, plus ou moins prêt à renaître : il reste bien une dualité virtuelle derrière chacune de ces positions ${ }^{10}$.

\section{UNE DYNAMIQUE DES POSITIONS}

Ces valeurs relatives des différentes positions ne sont que celles dans lesquelles les relations avec les non-humains s'achèvent ou, du moins, se stabilisent momentanément. Or, puisqu'elles se trouvent toujours dans les cas d'aflinité ou de consanguinité dédoublées entre deux positions simultanées, l'une actuelle et l'autre virtuelle, antérieures ou éventuelles, il ne faut pas les voir de manière statique, comme s'il s'agissait d'un cadre fixe et déjà donné, comme une société dans laquelle sujets humains et non humains seraient déjà socialisés, mais de manière dynamique, comme des passages et trajectoires entre plusieurs situations. Cela est particulièrement visible dans les cas mettant en scène des positions contradictoires, comme les activités cynégétiques des chamanes waiwai. Le chamane s'adressait au " père » des pécaris, lequel est un « ennemi », donc logiquement affinn, comme à un "grand-père ", sans doute pour établir une relation de protection et transmission de pouvoir avec celui-ci. Mais il nommait ensuite les pécaris « fils » - et non affins, en tant que gibier - comme pour les placer sous son contrôle et usurper la place du maître que les animaux nomment " père » (Fock 1963, pp. 26-30). Il semble donc que la puissance du chamane réside dans sa capacité à adopter différentes positions face aux non-humains, lesquelles sont de plus contradictoires entre elles. De même, Fausto a montré comment les relations avec les êtres du rêve chez les Parakanã, ordonnées en une séquence de « familiarisation " progressive, c'est-à-dire de consanguinité croissante depuis la position d'ennemi jusqu'à celle d'animal domestique, sont, une fois atteint ce degré ultime de la consanguinité, « réaffinisées » de manière contrôlée dans un cadre rituel au profit d'un autre humain qui obtient ainsi une « victime » (un chant) à exécuter - et donc un statut de tueur et de rêveur potentiel.

Les mécanismes de cette dynamique apparaissent cependant de manière plus précise lorsqu'on étudie le point de vue qui est attribué en retour à ces non- 
humains sur les humains. Cela est notamment visible dans un cas où la temporalité joue un rôle décisif : celui de ces ex-humains ou humains en voie de déshumanisation que sont les morts. On sait que les morts sont en général considérés comme des ennemis dont la mémoire doit être effacée ou tout au moins contrôlée. En tant qu'ennemis, ce sont donc des méta-aftins, qu'on accuse de chercher à séduire les vivants, mais chez qui certains pouvoirs peuvent parfois être obtenus (Viveiros de Castro 1992 ; Lima 1996). Néanmoins, le point de vue des morts est souvent aussi décrit comme tout autre. On rencontre certes quelques cas de perspectivisme absolument symétrique : les Kraho, par exemple, pensent que les morts voient les vivants comme des morts; chez les Miraña, les spectres, lorsqu'ils se manifestent dans les maisons des vivants, s'y comporte en « invités ", c'est-à-dire en affins (Carneiro da Cunha 1978 ; Karadimas 1997, p. 223). Les cas d'agressivité, conduite connotant l'aflinité, des morts à l'égard des vivants sont légion en Amazonie. Toutefois il y a de nombreux groupes où, à la différence des précédents, la perspective n'est pas entièrement symétrique. Ces eschatologies affirment en effet que certains morts, durant un certain temps ou dans certaines situations, perçoivent les vivants, non comme des ennemis ou des affins, mais comme des consanguins. Il est précisé que, tantôt, les morts voient les vivants comme « humains »-i.e. " vrais » humains, vivants, c'est le cas des Shipibo-, tantôt, les chants funéraires s'acharnent à nier leur relation de consanguinité, reconnaissant donc par là que les morts continuent à y prétendre - c'est le cas des Jivaro ou des Cashinahua (Leclerc 2003, p. 320 ; Taylor 1993b ; Kensinger 1995, p. 232). Ce qui se traduit comme une agressivité ou un désagrément pour les vivants peut-être attribué à un désir ou un refus d'oublier les liens de consanguinité. Même les Araweté, chez qui Viveiros de Castro (1992, p. 215) souligne l'extraordinaire développement du schème de l'affinité dans les relations avec les morts, avouent que les défunts ne perdent pas immédiatement le sens de la consanguinité : en effet, les morts récents ne participent pas aux festins cannibales célestes où dieux et morts anciens dévorent les nouveaux défunts, car " on ne saurait manger ses parents ». Les perspectives ne sont donc pas exactement inversées dans le cas de la relation entre vivants et morts ou, plus précisément, l'inversion y est moins rigoureuse qu'entre chasseur et proie. Or ces morts qui veulent voir les vivants comme des consanguins ont d'abord été des malades, des agonisants ou des séduits qui, certes, voyaient les morts comme des consanguins, mais surtout voyaient les vivants comme des étrangers, donc comme des affins. En effet, lorsque la séduction ou la maladie a atteint un stade avancé, le sujet reste sourd aux appels de ses parents, les rejette comme des étrangers, voire comme des esprits séducteurs. C'est le cas dans les attaques de l'entité noró chez les Yagua, dans celles du boráro chez les Desana, dans certains cas dans celles des démons kaniz chez les Candoshi, dans les maladies des Cashinahua ou des Yekuana (Chaumeil 2000, p. 174 ; Reichel-Dolmatoff 1975, p. 186 ; Guss 1980). La perspective semble alors s'être complètement renversée pour le sujet attaqué, mais pas 
pour les vivants qui veulent le sauver. Il semble donc que les positions s'engendrent dans une sorte de dialectique, chaque renversement de perspective en appelant un autre. En effet, ensuite, dans certains cas comme celui des Jivaro (Taylor 1993b), un mort sera l'objet d'une tentative de " reconsanguinisation », lors de la quête d'arutam par les vivants au moyen d'un langage affin (le chant anent), après avoir été préalablement " déconsanguinisé » contre son gré par ce même langage, lors du rituel d'oubli funéraire, alors qu'il s'était laissé auparavant « affiniser » contre leur volonté, lors d'une séduction entraînant la maladie, puis le décès.

Cette dialectique résulte du fait que les trois perspectives du sujet sur le non-humain, du sujet sur les humains et des humains sur le sujet ne coïncident jamais. Pour un cas purement « théorique » et reconstruit pour la trajectoire d'un individu masculin, on peut proposer le modèle suivant : un chasseur qui, croyant poursuivre un animal, succomberait à la séduction de l'esprit forestier que se révèlerait être cet animal, finirait comme une sorte d'animal familier de celui-ci, malgré les efforts de ses parents pour le sauver de la maladie, et serait finalement source de pouvoirs - il n'existe, bien entendu, aucun cas " pur » et « complet » comme celui-ci, relativement peu probable par ailleurs. Mais, en simplifiant quelque peu les positions et les trajectoires, il permet de voir que ces perspectives ne s'accordent quasiment jamais :

\begin{tabular}{|l|l|l|l|l|l|}
\cline { 2 - 6 } \multicolumn{1}{c|}{} & \multicolumn{1}{c|}{ Chasse } & $\begin{array}{l}\text { "Révélation } \\
\text { de l'esprit }\end{array}$ & $\begin{array}{c}\text { Séduction } \\
\text { achevée }\end{array}$ & $\begin{array}{c}\text { État de mort } \\
\text { " récent " }\end{array}$ & $\begin{array}{c}\text { État de mort } \\
\text { " ancien " }\end{array}$ \\
\hline $\begin{array}{l}\text { Non- } \\
\text { humain : } \\
\text { Sujet : }\end{array}$ & $\begin{array}{l}\text { Affine } \\
\text { séductrice }\end{array}$ & $\begin{array}{l}\text { Affin } \\
\text { dominant } \\
\text { (WF) }\end{array}$ & Maître (M / F) & $\begin{array}{l}\text { Consanguins } \\
\text { semblables (B) }\end{array}$ & $\begin{array}{l}\text { Consanguins } \\
\text { inférieurs (S) }\end{array}$ \\
\hline $\begin{array}{l}\text { Humains : } \\
\text { Sujet : }:\end{array}$ & Consanguins & $\begin{array}{l}\text { Affins ennemis } \\
\text { (WB) }\end{array}$ & $\begin{array}{l}\text { Affins ennemis } \\
\text { séducteurs }\end{array}$ & Consanguins & $\begin{array}{l}\text { Orphelins } \\
\text { (BS / ZS)/ } \\
\text { Gendres } \\
\text { faibles (DH) }\end{array}$ \\
\hline $\begin{array}{l}\text { Sujet : } \\
\text { Humains : : }\end{array}$ & Consanguins & Consanguins & Consanguins & $\begin{array}{l}\text { Affins / } \\
\text { Séducteurs }\end{array}$ & $\begin{array}{l}\text { Protecteur } \\
\text { (FF) }\end{array}$ \\
\hline
\end{tabular}

Si l'identité individuelle est déjà constamment sous la menace de sa définition relationnelle en Amazonie (Taylor 1996), lorsqu'il y a trois perspectives et non plus seulement deux en jeu, leur coïncidence et leur concordance deviennent nettement plus improbables, et l'identité du sujet au centre de ce conflit plus instable. Corrélativement, en répondant aux sollicitations des non-humains, le sujet humain entre généralement dans un champ de communication fragmentée où les diverses modalités de la parole humaine sont décomposées et dissociées - altération de la langue, dissociation des modalités sensorielles, perte des capacités de communication humaine... Simultanément, et par là même, la personne se fragmente elle-même en une multiplicité, sinon d'entités, du moins de points de 
vue - et cette dissociation signifie souvent la mort. L'entrée dans ce champ implique donc l'impossibilité d'une relation univoque et d'une position stable. Selon les termes de Viveiros de Castro (2001), l'espace entre les espèces ontologiquement distinctes, espace qui conserve des propriétés "pré-cosmologiques » - ou pré-mythiques - et qui est celui de la communication non humaine, est le lieu d'une « différence infinie et interne » : autrement dit, nul locuteur ne peut donc y avoir une position unifiée et non équivoque. Nous avons souligné cette dualité inhérente aux positions occupées par les non-humains; celle-ci se répercute désormais au sein de la personne de l'humain qui ose entrer dans une relation de communication avec eux.

Les interactions pourraient donc être qualifiées, mieux que par une seule position, par une succession d'au moins deux et associées à des déterminations corrélatives de la position du sujet humain par rapport aux autres humains. Chacune serait donc définie par une trajectoire, composée de plusieurs moments, ayant chacun leur propre valeur. Ainsi, par exemple, on peut laisser un nonhumain se présenter comme ascendant immédiat, lui laissant espérer une soumission, pour mieux l'affronter ensuite, afin de le réduire à un statut de descendant et donc d'auxiliaire soumis. Le caractère performatif des communications non humaines peut donc, dans cette perspective, être défini comme la capacité à savoir jouer de cet aspect équivoque et de cette dualité, pour la détourner à son profit. Le contenu des communications recouvre ainsi l'imposition par l'un des interlocuteurs à l'autre, d'une relation d'un certain type entre le sujet humain et le non-humain, et donc d'une position spécifique à ce dernier. Or ce qui permet d'enclencher ces dynamiques déterminant l'issue de l'interaction, ce sont toutes les « informations » qui s'y glissent dès l'amorce de ces dialogues, qu'il s'agisse des « propos » tenus, de leur style rhétorique, de l'aspect de l'interlocuteur ou du contexte de sa manifestation, dans la mesure où ces données rendent possibles ou induisent certaines relations - ainsi que leurs évolutions ou transformations - et en interdisent d'autres.

\section{Conclusion}

Apprendre un chant d'un tapir, séduire un pécari, rêver d'un mort ou être nourri par un esprit... Faits en apparence très hétéroclites, qui surgissent dans une multitude de croyances et de pratiques très diverses, sont donc des interactions qui relèveraient d'un même ensemble dans la mesure, du moins, où elles sembleraient pouvoir être analysées d'après un modèle dont les grandes lignes resteraient constantes à travers toutes les Basses Terres. Parmi ces traits récurrents, il y aurait l'usage d'un même principe interprétatif dans lequel les significations littérales et métaphoriques ne seraient que les pôles d'un continu. On compterait aussi l'emploi d'un même langage fondé sur la parenté et dont les termes sont 
construits à partir de la combinaison d'un petit nombre d'oppositions, les plus productives étant affīnité/consanguinité, identité/différence de génération, ascendance/descendance, ascendance lointaine/proche, identité/différence de sexe. Enfin, aucune de ces relations avec les non-humains ne peut être comprise isolément, séparément de l'ensemble des relations que le sujet entretient à la fois avec les humains et avec les non-humains : chaque interaction avec un nonhumain implique, entraine ou exclut un autre type de relations, présentes et à venir, avec ses parents humains et ses beaux-parents non-humains. *

* Manuscrit reçu en janvier 2005, accepté pour publication en mai 2005.

\section{NOTES}

1. Il n'y a guère de groupes chez qui l'existence de rêves "métaphoriques » est entièrement improbable. En effet si, chez certains, ce trait n'est pas mentionné par l'ethnographie, on le retrouve dans des groupes proches linguistiquement, culturellement ou géographiquement. Ainsi, par exemple, si de tels rêves ne semblent pas mentionnés chez nombre de groupes Tupi-Tapirapé, Araweté, Kaapor -, ils sont attestés chez d'autres comme les Mundurucu, Juruna et même, à un degré certes très faible - dans les présages de mort (Fausto 2001, p. 346) -, chez les Parakanã qui sont pourtant ceux qui insistent le plus sur le caractère « réel » et « littéral » des rêves. Il en va de même dans les groupes pano ou gé. En revanche, nous ne pouvons mentionner de cas où des rêves ne seraient pas considérés comme des interactions « réelles » ou comme ayant des conséquences immédiatement « réelles 》. Notons que ces deux classes sont parfois nommées, selon une terminologie piercéenne, « iconiques » et « indiciaires ", la seconde catégorie permettant de regrouper rêves littéraux et rêves " insignifiants » qui, dans des groupes comme les Kalapalo ou les Wayana (Basso 1987 ; Chapuis 1998), ne sont que des souvenirs de la veille, en tant qu'ils constituent des résidus d'actes réels.

2. Hypothèse intéressante, car on peut y retrouver un principe fondamental de la communication non humaine en Amazonie : celui de proportionnalité inverse entre les capacités de communication (simultanée) dans les champs non humain et humain. Ce serait parce qu'on peut les rendre publics que certains messages ont une forme métaphorique afin de maintenir un écart entre ces deux sphères de communication.

3. Les mythes d'origine du rêve sont extrêmement rares en Amazonie, ce qui n'est pas surprenant si on considère que le mythe raconte précisément la fin de l'ère pré-cosmologique où humains et non-humains communiquaient librement, situation qui est réactualisée par le rêve - «littéral » du moins - partiellement et momentanément. Un mythe «d'origine » du rêve paraît donc, sinon une contradiction dans les termes, du moins un mythe sur la limite de la mythologie - de la césure entre l'univers pré-cosmologique et le monde actuel différencié en espèces. Il existe néanmoins un mythe de cette sorte chez les Trio (Magaña 1990). Il y est question de l'origine des rêves "prophétiques " qui nécessitent toujours une interprétation "métaphorique ». Une analyse superficielle montre que les deux versions recueillies semblent insister sur la différence de degré entre les événements du rêve et la réalité. La première donne une étiologie « indiciaire », mais affaiblie des contenus oniriques : Sarigue "mime une attaque " sur le rêveur qui la rêve. La seconde croise le thème courant du "mauvais choix " : l'homme, ayant choisi le rêve, se voit privé de l'abondance inépuisable de viande qui se reconstituerait sur les os du gibier cuit; le mauvais choix serait donc ici celui d'une relation épisodique, incertaine et difficile avec le gibier, excluant une relation constamment et automatiquement actualisée. 
4. Il faudrait sans doute retenir également l'écart éventuel entre les termes d'adresse - du moins dans les cas où ils sont rapportés ou dans ceux où l'interaction est observable (publique) - et les termes de référence dans les récits ou descriptions. Malheureusement, à l'exception de très rares cas, les données explicites sont lacunaires sur ce point. Précisons que, par commodité, nous avons classé les positions en fonction d'un Ego humain et, sauf mention particulière, masculin.

5. Cette dualité est un schème récurrent qui se retrouve au niveau de chaque non-humain. C'est du moins le cas des animaux dont une partie est souvent appropriable, ou consommable, tandis qu'une autre, réelle ou immatérielle, doit être abandonnée pour permettre leur renouvellement et leur conservation (Stephen Hugh-Jones 1996 ; Taylor 2000).

6. Dans la terminologie dravidienne, l'opposition consanguin/affin ne s'applique plus aux grandsparents. Ce ne sont donc pas à proprement parler des consanguins, mais ce ne sont pas non plus des affins, ni réels, ni virtuels, ni des méta-aftins.

7. Il serait intéressant, comme me l'a suggéré Olivier Allard (communication personnelle), de tenter une comparaison entre ces consanguinisations en position d'ascendants lointains d'entités méta-affines et l'aflinisation "réelle », par un rapport d'alliance, d'anciens ascendants en train de se méta-affiniser (morts "vieillissants ») mise au jour chez les Araweté par Viveiros de Castro.

8. Nous n'incluons pas les cas où il s'agit d'une théorie générale de la conception, comme chez les Tapirapé, pour qui tout enfant doit être produit par le vol d'une identité chez les animaux (Wagley 1977). Dans ce cas, la qualification de non humain parmi les enfants n'est bien sûr plus pertinente.

9. Sauf dans un cas complexe, chez les Karaja, des esprits généralement nommés " grands-pères » et jouant un rôle « protecteurs et nourriciers » sont parfois appelés " pères » (Pétesch 1987).

10. Parmi ces diverses formes de "consanguinisation ", une absence est remarquable : celle des positions en $\mathrm{G} 0$. On pourrait peut-être en voir une forme, intermédiaire et cumulative de l'aftinité et de la consanguinité, dans les positions d'« ami cérémoniel » qui sont parfois attribuées ou imposées aux esprits de victimes d'homicide, par exemple chez les Araweté ou chez les Nivacle du Chaco (Viveiros de Castro 1992, p. 240 ; Sterpin 1993). Une position de germain de même sexe n'aurait peut-être guère de sens ou d'intérêt car trop marquée par la rivalité - le seul exemple connu de nous, imprécis, dans un rêve siona, est clairement hypocrite, mensonger et malveillant (Langdon 1999, p. 43) - ou trop contradictoire avec l'origine non humaine. Il est même peut-être envisageable qu'elle soit impossible car déjà donnée sous une forme maximale pour être immédiatement supprimée. Car chaque Amazonien naît avec un jumeau non humain, dont le développement doit être à tout prix empêché pour permettre le sien et pour éviter les pires catastrophes cosmologiques : c'est son placenta (Viveiros de Castro 2001 ; Christine Hugh-Jones 1979, p. 118). Or, à notre connaissance, nul ne communique avec un placenta ou, plus exactement, avec son propre placenta, puisqu'il semble possible d'apercevoir, dans plusieurs cas, une association complexe entre placentas, certains palmiers - entre autres Bactris et/ou Mauritia qui sont réputés pour avoir des esprits puissants et grands communicateurs par les Yagua, et dont les premiers sont nommés «nos placentas », tandis que les seconds sont nourris de bière de manioc -, certains instruments rituels (flutes yagua, masques matis) associés à un rôle initiateur, maturateur et/ou protecteur, et « ancestralité » (Chaumeil 2001 ; Erikson 1996). Les placentas avec lesquels on interagirait seraient donc ceux de morts anciens - ou devenus eux-mêmes des morts anciens -, dans une position oscillant entre ascendance lointaine et affinité ou amitié cérémonielle lointaine - étrangère (Erikson 2004). 


\section{BIBLIOGRAPHIE}

Albert Bruce

1985 Temps du sang, temps des cendres : représentation de la maladie, système rituel et espace politique chez les Yanomami du sud-est (Amazonie brésilienne), thèse de doctorat, université Paris X, Nanterre.

ARHEM Kaj

1996 "The cosmic food web : human-nature relatedness in the Northwest Amazon ", in P. Descola et G. Pálsson, éds, Nature and society : anthropological perspectives, Routledge, Londres, pp. 185-204.

BAER Gerhard

1992 "The one intoxicated by tobacco : Matsigenka shamanism », in E. J. M. Langdon et G. Baer, éds, Portals of power : shamanism in South America, University of New Mexico Press, Albuquerque, pp. 79-100.

Basso Ellen B.

1987 "The implicaions of a progressive theory of dreaming », in B. Tedlock, Dreaming : anthropological and psychological interpretations, Cambridge University Press, Cambridge, New York, pp. 86-104.

BIRD-DAVID Nurit

1999 " "Animism" revisited : personhood, environment and relational epistemology », Current Anthropology, 40, pp. 67-91.

BRown Michael F.

1985 Tsewa's gift: magic and meaning in an Amazonian society, Smithsonian Institution Press, Washington.

CARNEIRo Robert L.

1964 "The Amahuaca and the Spirit World », Ethnology, 3 (1), pp. 6-11.

CARneiro da CunHa Manuela

1978 Os Mortos e os Outros : uma análise do sistema funerário et da noção de pessoa entre os indios Krahó, Hucitec, São Paulo.

Chapuis Jean

1998 La personne wayana : entre sang et ciel, thèse de doctorat, université d'AixMarseille, Aix-en-Provence.

ChaumeIL Jean-Pierre

2000 Voir, savoir, pouvoir : le chamanisme chez les Yagua de l'Amazonie péruvienne, Georg, Genève [1983].

2001 "The blowpipe Indians : variations on the theme of blowpipe and tube among the Yagua Indians of the Peruvian Amazon ", in L. Rival et N. Whitehead, éds, Beyond the Visible and the Material : the amerindianization of society in the work of Peter Rivière, Oxford University Press, Oxford, pp. 81-99. 


\section{COFFACI de Lima Edilene}

2000 Com os Olhos da Serpente: homens, animais e espiritos nas concepções katukina sobre a natureza, thèse de doctorat, universidade de São Paulo, São Paulo.

\section{CRÉPEAU Robert R.}

2000 "La pratique du chamanisme chez les Kaingang du Brésil méridional », in D. Aigle, B. Brac de la Perrière et J.-P. Chaumeil, éds, La politique des esprits, Société d'ethnologie, Nanterre, pp. 309-322.

Crocker Jon Christopher

1985 Vital Souls : Bororo cosmology, natural symbolism and shamanism, University of Arizona Press, Tucson.

\section{Da Matta Roberto}

1970 "Les présages apinayé », in J. Pouillon et P. Maranda, éds, Échanges et communications : mélanges offerts à Claude Lévi-Strauss, Mouton, Paris, pp. 77-99.

Descola Philippe

1989 « Head-shrinkers versus shrinks : Jivaroan analysis of dream », Man, n.s., 24 (3), pp. 439-450.

1992 "Societies of nature and the nature of society », in A. Kuper, éd., Conceptualizing Society, Routledge, Londres, pp. 107-126.

1993 Les lances du crépuscule, Plon, Paris.

1994 "Pourquoi les Indiens d'Amazonie n'ont-ils pas domestiqué le pécari ? ", in B. Latour et P. Lemonnier, éds, De la préhistoire aux missiles balistiques, La Découverte, Paris, pp. 329-344.

DESHAYES Patrick

1992 "Paroles chassées : chamanisme et chefferie chez les Kashinawa », Journal de la Société des Américanistes, 78 (2), pp. 95-106.

ERIKSON Philippe

1996 La griffe des aïeux, Peeters/Selaf, Louvain/Paris.

2004 «La face cachée de l'ancestralité », Journal de la Société des Américanistes, 90 (1), pp. 119-142.

\section{Fausto Carlos}

1999 "Of enemies and pets : warfare and shamanism in Amazonia ", American Ethnologist, 26 (4), pp. 933-956.

2001 Inimigos Fiéis : história, guerra e xamanismo na Amazônia, EdusP, São Paulo.

Fock Niels

1963 Waiwai. Religion and Society of an Amazonian Tribe, Nationalmuseets Skrifter, Copenhague.

Graham Laura

1995 Performing dreams : discourses of immortality among the Xavante of Central Brazil, University of Texas Press, Austin. 
GrEgOr Thomas

1977 Mehinaku. The Drama of Daily Life in a Brazilian Indian Village, The University of Chicago Press, Chicago.

Guss David M.

1980 « Steering for dream : dream concepts of the Makiritare », Journal of Latin American Lore, 6 (2), pp. 297-312.

Henry Jules

1964 Jungle People : a Kaingáng tribe of the Highlands of Brazil, Vintage Books, New York [1941].

Howard Catherine V.

2001 Wrought identities : the Waiwai expeditions in search of the "Unseen Tribes » of Northern Amazonia, thèse de doctorat, University of Chicago, Chicago.

Hugh-Jones Christine

1979 From the Milk River : spatial and temporal processes in Northwest Amazonia, Cambridge University Press, Cambridge.

Hugh-JoNes Stephen

1996 «Bonnes raisons ou mauvaise conscience? », Terrain, 26, pp. 123-148.

KaRADimas Dimitri

1997 Le corps sauvage : idéologie du corps et représentations de l'environnement chez les Miraña d'Amazonie colombienne, thèse de doctorat, université Paris X, Nanterre.

KeIFENheim Barbara

2002 «Suicide à la kashinawa : le désir de l'au-delà ou la séduction olfactive par les esprits des morts ", Journal de la Société des Américanistes, 88, pp. 91110.

Kensinger Kenneth M.

1995 How Real People Ought to Live : the Cashinahua of Eastern Peru, Waveland Press, Prospect Heights, Ill.

KRACKE Waud H.

1990 «El sueño como vehículo del poder shamánico : interpretaciones culturales y significados personales de los sueños entre los parintintin "), in M. Perrin, éd., Antropología y experiencias del sueño, Abya-Yala, Quito, pp. 145-157.

LANGDON Esther Jean

1999 "Representações do poder xamanístico nas narrativas dos sonhos Siona ", Ilha. Revista de antropologia, 1, pp. 35-56.

LECLERC Frédérique Rama

2003 Desmodes de socialisation par les plantes chez les Shipibo-Conibo d'Amazonie péruvienne, thèse de doctorat, université Paris X, Nanterre.

Lıma Tânia Stolze

1996 «Os dois e seu múltiplo : reflexões sobre o perspectivismo em uma cosmologia tupi », Mana, 2 (2), pp. 21-47. 
MaGaÑa Edmundo

1990 "Zariguëya, señor de los sueños : una teoría tarëno ", in M. Perrin, éd., Antropología y experiencias del sueño, Abya-Yala, Quito, pp. 117-143.

MURPhy Robert F.

1958 Mundurucú Religion, University of California Press, Berkeley.

PASSES Allan

1998 The hearer, the humter and the agouti head: aspects of intercommunication and conviviality among the Pa'ikewené (Palikur) of French Guiana, thèse de doctorat, University of St Andrew, Fife.

Petesch Nathalie

1987 «Divinités statiques, hommes en mouvement : structure et dynamique cosmique et sociale chez les Indiens Karajá du Brésil central ", Journal de la Société des Américanistes, 73, pp. 75-92.

Reichel-DolmatofF Gerardo

1973 Desana. Le symbolisme universel des Indiens Tukano du Vaupès, Gallimard, Paris.

1975 The shaman and the jaguar : a study of narcotic drug among the Indians of Colombia, Temple University Press, Philadelphie.

Rival Laura

1996 «Blowpipes and spears : the social significance of Huaorani technological choices "), in P. Descola et G. Pálsson, éds, Nature and society : anthropological perspectives, Routledge, Londres, pp. 145-164.

SALAdin D'Anglure Bernard et Françoise Morin

1998 "Mariage mystique et pouvoir chamanique chez les Shipibo d'Amazonie péruvienne et les Inuit du Nunavut canadien ", Anthropologie et sociétés, 22 (2), pp. 49-74.

SeEger Anthony

1981 Nature and society in Central Brazil : the Suya of Mato Grosso, Harvard University Press, Cambridge, Mass.

Sperber Dan et Wilson Deirdre

1989 La pertinence : communication et cognition, Minuit, Paris.

STERPIN Adriana

1993 "La chasse aux scalps chez les Nivacle du Gran Chaco », Journal de la Société des Américanistes, 79, pp. 33-66.

SurRallés Alexandre

2003 Au cour du sens. Perception, affectivité et action chez les Candoshi, CNRS Éditions, Paris.

TAYlor Anne-Christine

1993a "Des fantômes stupéfiants : langage et croyance dans la pensée achuar », L'Homme, 126-128, pp. 429-447.

1993b «Remembering to forget: identity, mourning and memory among the Jivaro ", Man, n.s., 28 (4), pp. 653-678. 
1996 "The soul's body and its states : an Amazonian perspective on the nature of being human ", Journal of the Royal Anthropological Institute, n.s., 2 (2), pp. 201-215.

2000 "Le sexe de la proie : représentations jivaro du lien de parenté », L'Homme, 154-155, pp. 309-334.

VILAÇA Aparecida

1999 «Devenir autre : chamanisme et contact interethnique en Amazonie brésilienne ", Journal de la Société des Américanistes, 85, pp. 239-280.

2002 " Making kin out of others 》, Journal of the Royal Anthropological Institute, n.s., 8 (2), pp. 347-365.

Viviros de Castro Eduardo

1992 From the enemy's point of view, The University of Chicago Press, Chicago.

1998 "Cosmological deixis and Amerindian perspectivism", Journal of the Royal Anthropological Institute, n.s., 4 (3), pp. 469-488.

2001 "GUT feelings about Amazonia : potential affinity and the construction of sociality ", in L. Rival et N. Whitehead, Beyond the visible and the material : the amerindianization of society in the work of Peter Rivière, Oxford University Press, Oxford, pp. 19-43.

WAGLEY Charles

1977 Welcome of tears. The Tapirapé Indians of Central Brazil, Oxford University Press, New York.

WILBERT Johannes

1993 Mystic endowment. Religious ethnography of the Warao Indians, Harvard University Press, Cambridge, Mass. 